\title{
A Seamless Handover for WSN Using LMS Filter
}

\author{
Waltenegus Dargie and Jianjun Wen
}

\author{
Chair for Computer Networks, Faculty of Computer Science, Technical University of Dresden, 01062 Dresden, Germany \\ Email:\{waltenegus.dargie, jianjun.wen\}@tu-dresden.de
}

\begin{abstract}
We propose a MAC protocol that supports the mobility of nodes in wireless sensor networks. The protocol enables burst transmission and seamless handover to achieve high throughput and to reduce packet delivery latency and packet loss. An adaptive filter continuously evaluates the RSSI values of received acknowledgment packets and decides whether a mobile node should transfer a communication to a nearby relay node with a better link quality. The handover process itself takes place without breaking an existing link. This paper presents the design, implementation and evaluation of the MAC protocol.

Index Terms-Arthroplasty, Biotelemetry, MAC protocol, medical applications, mobility, wireless sensor networks
\end{abstract}

\section{INTRODUCTION}

Wireless sensor networks enabling the free movement of sensing nodes [1] are useful for many applications, for example, for supervising post-surgery rehabilitation after total hip and knee replacement (Arthroplasty) [2] and Biotelemetry [3]. During rehabilitation, the progress of a patient can be measured by evaluating gait velocity, stride length, single-leg stance time, step width, peak hip abduction at early and late stance, internal and external rotation, and hip and knee flexion and extension. Similarly, in Biotelemetry patients can be free to carry out everyday activities while their heart rate and brain activities are monitored by wireless Electrocardiograph (ECG) and Electroencephalograph (EEG).

Unlike applications which require a static deployment and low packet delivery rate, applications with mobile sensor nodes require high data quality [4]. However, during mobility the quality of an established link between a mobile transmitter and a stationary relay node greatly fluctuates resulting in high packet loss, end-to-end latency, and irregular packet arrival time (jitter in packet arrival time). Fig. 11 compares the fluctuations of link quality while a mobile node transmits packets in burst. In the figure, the mobile node first located at $4,8,30$, and $40 \mathrm{~m}$ away from the static relay node moved within an additional distance of $4 \mathrm{~m}$ while transmitting packets at $250 \mathrm{Kbps}$. The receiver node acknowledged each successfully received packets. The figure displays the RSSI values of the acknowledgment packets and illustrates how the link quality between the two communicating nodes fluctuates. In addition, mobility introduces high packet loss compared with communication without mobility. We shall give a report about the packet loss of different scenarios in Section IV]

In this paper, we propose a MAC protocol to assist the free mobility of nodes. We shall experimentally show that it achieves high packet delivery rate, low latency, and low packet loss. Its essential features are dynamic link quality estimation and seamless handover. The remaining part of the paper is organized as follows: In Section III] we present our approach conceptually. In Section III we provide a detail account of the implementation of our protocol. In Section IV] we provide a quantitative evaluation of our protocol. Finally, in Section $\mathrm{V}$ we provide concluding remarks.

\section{APPROACH}

Whether in a home or a clinical environment, it is possible to deploy stationary relay nodes to assist mobile nodes. The communication link between the relay nodes and the remote base station(s) can be stable, as the topology of the static nodes does not need to change frequently. Therefore, it is sufficient to address the challenges surrounding a singlehop link connecting the mobile nodes with the relay nodes, which is the task of the link layer protoco Hence, fast medium access, burst transmission, and seamless handover can fulfill the requirements of mobile applications and address the challenges associated with mobility.

1) Bulk Data Transfer: In most existing contention-based MAC protocols nodes transmit each packet after wining the medium through contention. As long as the packet generation rate of a node is low, these protocols are sufficient, but when the packet generation rate becomes high, the throughput of these protocols becomes a significant bottleneck. Recently, a new batch of MAC protocols has been proposed to enable bulk data transfer, and, thereby, achieve high throughput [5], [6]. These protocols enable node to transmit packets in a burst once they win a free channel.

2) Handover: A seamless handover (or hand-off) is one of the key features of cellular networks because it enables a mobile node to maintain an uninterrupted communication with a remote partner even though it changes a number of base stations on the way. In wireless sensor networks, a seamless handover can be very useful for transferring bulk data as well as for enabling live monitoring. In cellular networks, a handover process relies on the existence of a wired infrastructure with sufficient resources. Unfortunately, the relay nodes in wireless sensor networks are in every respect similar to the mobile nodes and cannot handle a handover process on their own. This requires a distributed and lightweight handover mechanism that takes the limitations of wireless sensor networks into account.

${ }^{1}$ It is widely accepted that the predominant traffic flow in wireless sensor networks is from sensing nodes to a base station. 

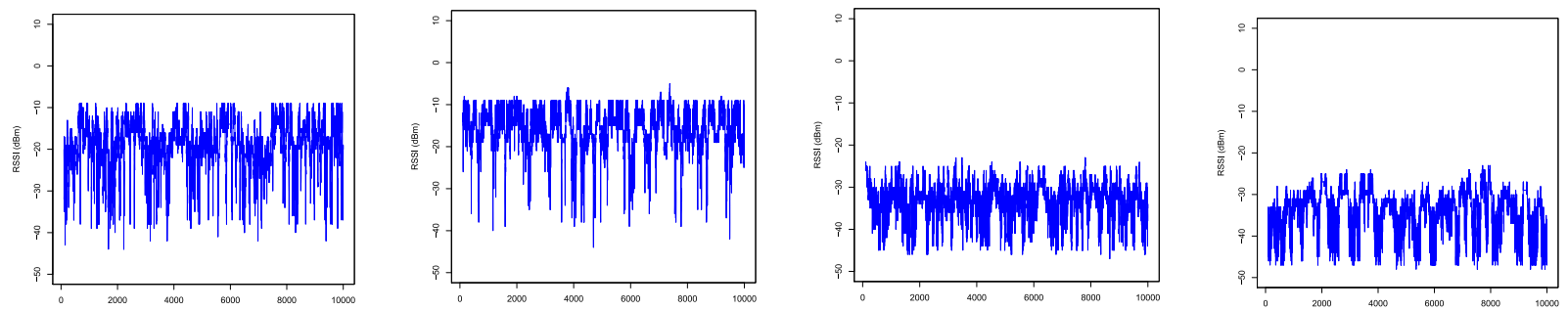

Fig. 1: The fluctuation of RSSI values of acknowledgment packets when the transmitter was mobile. The mobility range was $4 \mathrm{~m}$ and the nominal separation distance was (from left to right): $4 \mathrm{~m}, 8 \mathrm{~m}, 30 \mathrm{~m}$, and $40 \mathrm{~m}$.

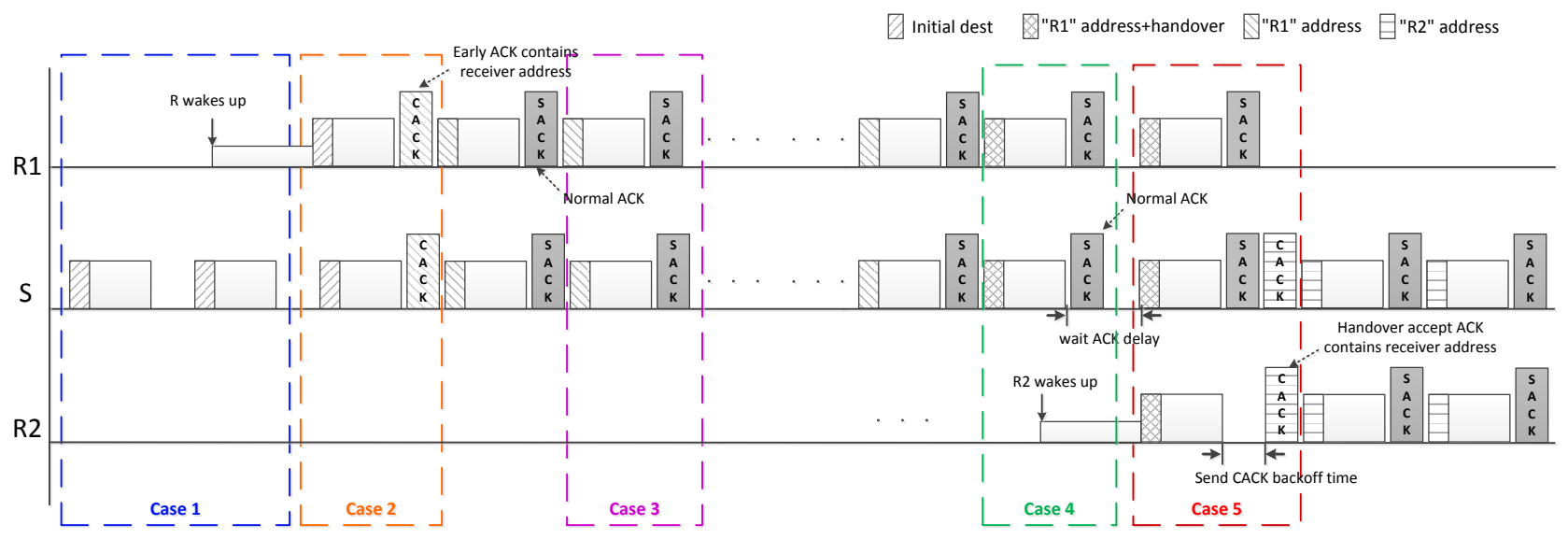

Fig. 2: A detail description of the different steps of a handover process.

Conceptually, a mobile node can initiate a handover process whenever the quality of an existing link drops below a set threshold for a long time. The quality of a link can be evaluated using the RSSI values of incoming acknowledgment packets. One approach to establish a new link without breaking the existing one is to send out a handover request to nearby relay nodes without breaking an existing link. This can be done by embedding a handover request in outgoing data packets. We define a 16 bit addressing mechanism to enable mobile nodes to discover relay nodes and to communicate with them seamlessly. The most significant bit (the 15-th bit) is reserved for a handover. By flagging this bit to 1 , a mobile node expresses a wish for a handover. The second most significant bit expresses the node type -1 is reserved for relay nodes and 0 is reserved for mobile nodes. Hence, relay nodes need only evaluate the address header to participate in a handover request. Fig. 2 shows the detail steps to support a seamless handover in a mobile environment. In the beginning, a mobile node wins a medium after contention and sends preamble to express interest in a communication (Case 1). A nearby relay node responds to the preamble by embedding its own address in the acknowledgment packet (Case 2). A burst communication follows with each packet acknowledged by the relay node, but this time without embedding an address (Case 3). All other nodes in the vicinity refrain from contending to win the medium, until the burst transmission is completed.
If the link quality deteriorates for sometime, then the mobile node flags the handover bit to 1 and continues communicating with the relay node (Case 4). The handover flag reveals the intention of the mobile node to the original relay node as well as to the surrounding relay nodes which intercept the outgoing packets from the mobile node. If the link quality of the surrounding relay nodes is better than the old link, then the mobile node chooses one of them, flags down the handover bit to 0 and communicates with the new relay node using a unicast address (case 5).

3) Link Quality Estimation: There is a link quality threshold below which the transmission of packets results in considerable packet loss or latency (due to retransmission of lost packets). For a CC2024 radio, packet loss rate drastically increases once the RSSI values of received packets drops approximately below $-85 \mathrm{dBm}$ [7], [8]. Unfortunately, the RSSI values of received packets may cross this threshold even when there is no mobility, though the fluctuation due to mobility is dominant (see Fig. 1). Hence, the success of a handover depends on the existence of a reliable link quality estimation model to determine when the handover should take place and the availability of relay nodes in the vicinity of the mobile node. Because it is difficult to predict how future RSSI values fluctuate, it is reasonable to model them as a discrete stochastic process $r[n]$. The advantage of this approach is that it is possible to reason about the link quality deterioration without the need to directly deal with its causes (such as 
placement of nodes on the mobile object, speed of mobility, or communication distance [9]). In which case, an adaptive finite impulse response filter can be used to predict the future values of $r[n]$ and the duration below which $r[n]$ remains below a set threshold. Thus,

$$
r_{p}[n]=\sum_{k=1}^{N} h_{n}[k] r[n-k]
$$

where $N$ is the number of packets required for prediction, $r[n-1]$ is the actually measured RSSI value at the time instance $n-1$, and $h_{n}[k]$ is the $k$-th coefficient of the filter for the $n$-th round. The filter's coefficients are dynamically updated. For any arbitrary $\alpha, 0<\alpha<1$, the predicted RSSI value for the $n+1$ packet can be expressed as:

$$
r_{p}[n+1]=\alpha r[n]+(1-\alpha) r_{p}[n]
$$

where $r_{p}[n]$ is the predicted RSSI value of the $n$th received acknowledgment packet (before it was received). Alternatively, Equation 2 can be rewritten as:

$$
r_{p}[n+1]=r_{p}[n]+\alpha\left(r[n]-r_{p}[n]\right)
$$

The expected error between the predicted and the actual RSSI values (the second term in Equation 3 determines the value of $\alpha$. If this error is small (i.e., if the RSSI values are highly correlated), then we trust the prediction and, therefore, make $\alpha$ deliberately small. If, on the other hand, the expected error is large, we put little trust on the prediction value and, therefore, make $\alpha$ large. In general, the error can be modeled as a normally distributed random variable with zero mean and $\sigma^{2}$ variance.

4) Duration Estimation: A handover request potentially introduces two types of latencies. The first is introduced when the mobile node searches for neighbor relay nodes. This cost is inevitable if a handover process is initiated. The second cost is introduced if a mobile node keeps on sending neighbor discovery requests but does not discover any relay node in its surrounding. The second type of cost is not limited to a handover process but can also occur if the link is disconnected and the mobile node has to establish a new link with a new relay node. The first cost can be high if the mobile node initiates a handover request often, for example, if a mobile node operates at the receiving boundary of two or more relay nodes (we call this phenomenon a handover oscillation). To reduce the cost of a handover, a mobile node should ensure that the deterioration in the link quality is a persistent condition. If $p$ refers to the probability that the RSSI value of an incoming acknowledgment packet drops blow $r_{t h}$, then, the expected number of packets whose RSSI values persistently drops below $r_{t h}$ can be expressed in terms of $p$ :

$$
E[d \mid p]=\sum_{k=1}^{\infty} k p^{k}(1-p)=p(1-p) \frac{d}{d p} \sum_{k=1}^{\infty} p^{k}=\frac{p}{(1-p)}
$$

Handover is feasible if $E[d \mid p]>t_{t h}$ and $t_{t h}$ is application specific.

\section{IMPLEMENTATION}

We implemented and integrated our protocol with the IMote2 sensor platform by extending the TinyOS Low Power Listening (LPL) package [10]. Thus, when there is no handover, our protocol acts similar to a preamble- and contentionbased protocol. We included three additional components to the LPL layer, two of them (the RSSI Partitioner and the Adaptive Filter) are used to predict link quality and one of them (Handover Decision Support) is used to initiate a handover.

Similar to the phenomena of slow and fast fading in mobile communications, the RSSI values of a mobile node experience fast and slow fluctuations (see Fig. 1). The fast fluctuations depend on factors such as placement, mobility type, and surrounding objects. For a human being taking a normal walk in an outdoor environment, this fluctuation is usually contained within $\pm 10 \mathrm{mdB}$. For an indoor environment, it can be more than this. The second type of fluctuation depends on the distance between the transmitter and the receiver and on the path loss index. Unless significant multi-path and shadowing components exist, distance plays the dominant role for this type of fluctuation. For a CC2420 radio, almost all packets can be successfully received if the quality of a link is above $-85 \mathrm{dBm}$ (unscaled). To predict the RSSI values of future acknowledgment packets, we divide the fluctuation range $(0 \leq r \leq 100 \mathrm{dBm})$ by the maximum magnitude of the fast fading component (i.e., $20 \mathrm{dBm}$ ). This results in five RSSI quality zones. The RSSI partitioning component assigns the raw RSSI values of incoming acknowledgment packets into one of these zones.

We implemented a Least Mean Square filter with depth 5 to predict to which of the five zones future incoming acknowledgment packets belong (it requires 17 multiplications and 15 additions for each prediction). The coefficients of the adaptive filter are dynamically updated by taking the difference between the predicted and the actually measured RSSI values into consideration. The number of samples the filter takes into consideration is configurable, depending on the environment in which the mobile node operates. We took 10 samples for our experiment. The handover decision support takes the input of the adaptive filter and determines if a handover initiation is necessary (using Equation 44. In addition, this component is responsible for embedding neighbor discovery request in outgoing packets and for selecting the best alternative relay node.

\section{Evaluation}

We evaluated the protocol in terms of the success of handover (to verify that the protocol functions as it is specified) and packet loss. We considered both indoor and outdoor environments and carried out several experiments, but in this paper we present the results pertaining to the outdoor experiments. In all the scenarios the task is to transfer 100000 packets from a mobile transmitter to a stationary relay node. The mobile node moved approximately at a speed of $1.5 \mathrm{~m} / \mathrm{s}$. 
Fig. 3 demonstrates the communication transfer during a handover. A mobile node moved between two relay nodes while transmitting packets in burst. Initially it was near R1 but gradually it moved away from it and towards R2 without stopping or changing its direction. The mobile node successfully transmitted the packets to a base station which was twohops away from it. During transmission, it changed relay nodes seamlessly.

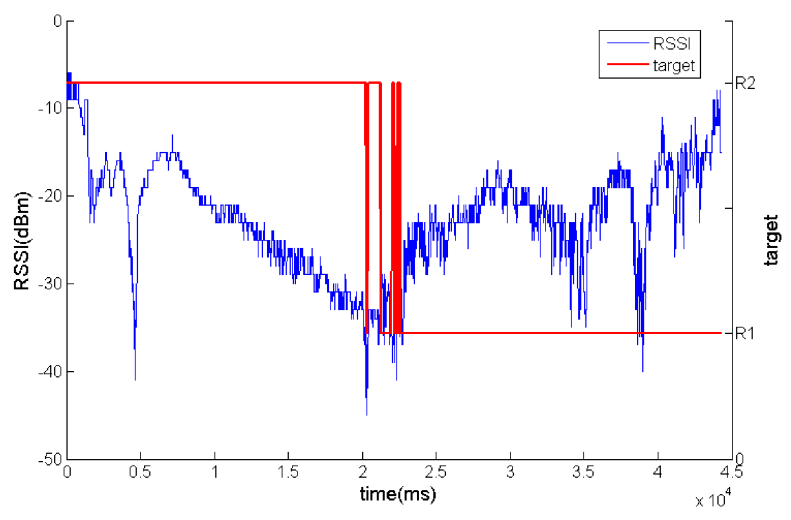

Fig. 3: Handover between two relay nodes.

Fig. 4 displays the packet loss experienced by the mobile node in different scenarios. Four of the scenarios are those displayed in Fig. 1 in which the nominal distance between the transmitter and one of the relay nodes was 4, 8, 30, and $40 \mathrm{~m}$ but the transmitter moved within an additional distance of $+4 \mathrm{~m}$ while transmitting. No handover mechanism was implemented. In the fifth scenario, the transmitter and the receiver were together initially but the transmitter moved steadily away from the receiver and covered a distance of 55 $\mathrm{m}$ while transmitting packets in burst. Here as well, there was no handover mechanism implemented. Finally, in the sixth scenario which is similar to the fifth scenario, we implemented a handover mechanism and placed an additional relay node approximately $30 \mathrm{~m}$ away from the first relay node. The mobile node started transmission from $10 \mathrm{~m}$ away from the first relay node and moved towards the second relay node and completed

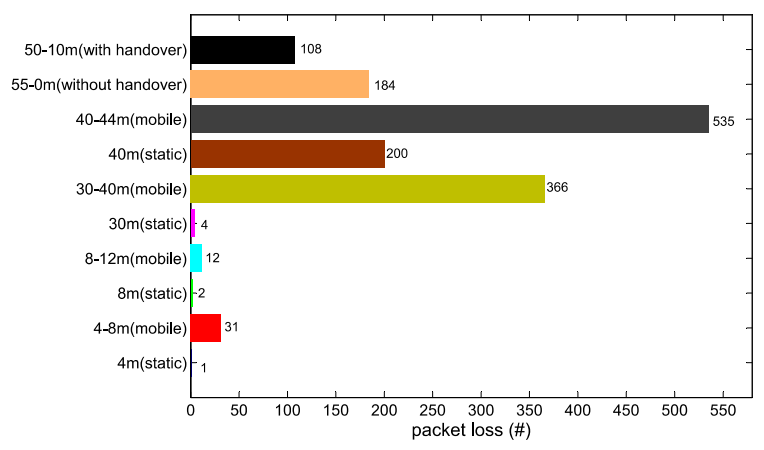

Fig. 4: Comparison of packets loss in different scenarios. transmitting the 10000 packets when it was $50 \mathrm{~m}$ away from the first relay node. During this time, it initiated a handover. As can be seen from the figure, a handover enabled the mobile node (1) to complete the burst transmission more quickly and (2) to reduce the packet loss.

\section{CONCLUSION}

In this paper we proposed a MAC protocol for wireless sensor networks that enable the free movement of some nodes. The protocol enables burst transmission as well as a seamless handover to achieve high throughput and live monitoring. We implemented the protocol for TinyOS in an IMote2 platform. We also carried out repeated experiments and observed that the seamless handover reduces single-hop latency and packet loss. In order to reduce the handover latency of burst transmission, the number of mobile nodes should be small. This, however, is typically the case for many health care applications in home and clinical settings.

\section{ACKNOWLEDGMENT}

This work has been partially funded by the German Research Foundation under project agreement: DA 1211/5-1.

\section{REFERENCES}

[1] W. Dargie and T. Tersch, "Recognition of complex settings by aggregating atomic scenes," Intelligent Systems, IEEE, vol. 23, pp. 58-65, Sept 2008.

[2] A. Bhave, D. R. Marker, T. M. Seyler, S. D. Ulrich, J. F. Plate, and M. A. Mont, "Functional problems and treatment solutions after total hip arthroplasty," The Journal of Arthroplasty, vol. 22, no. 6, Supplement, pp. $116-124,2007$

[3] J. G. Webster, Medical Instrumentation Application and Design, 4th Edition. Wiley Publishing, 2009.

[4] K. Lorincz, B.-r. Chen, G. W. Challen, A. R. Chowdhury, S. Patel, P. Bonato, and M. Welsh, "Mercury: a wearable sensor network platform for high-fidelity motion analysis," in Proceedings of the 7th ACM Conference on Embedded Networked Sensor Systems, SenSys '09, (New York, NY, USA), pp. 183-196, ACM, 2009.

[5] S. Duquennoy, F. Österlind, and A. Dunkels, "Lossy links, low power, high throughput," in Proceedings of the 9th ACM Conference on Embedded Networked Sensor Systems, SenSys '11, (New York, NY, USA), pp. 12-25, ACM, 2011.

[6] S. Kim, R. Fonseca, P. Dutta, A. Tavakoli, D. Culler, P. Levis, S. Shenker, and I. Stoica, "Flush: A reliable bulk transport protocol for multihop wireless networks," in Proceedings of the 5th International Conference on Embedded Networked Sensor Systems, SenSys '07, (New York, NY, USA), pp. 351-365, ACM, 2007.

[7] K. Srinivasan, M. A. Kazandjieva, S. Agarwal, and P. Levis, "The $\beta$ factor: Measuring wireless link burstiness," in Proceedings of the 6th ACM Conference on Embedded Network Sensor Systems, SenSys '08, (New York, NY, USA), pp. 29-42, ACM, 2008.

[8] W. Dargie, "A medium access control protocol that supports a seamless handover in wireless sensor networks," J. Netw. Comput. Appl., vol. 35 , pp. 778-786, Mar. 2012.

[9] W. Dargie, "Analysis of time and frequency domain features of accelerometer measurements," in Computer Communications and Networks, 2009. ICCCN 2009. Proceedings of 18th Internatonal Conference on, pp. 1-6, Aug 2009.

[10] K. Klues, G. Hackmann, O. Chipara, and C. Lu, "A componentbased architecture for power-efficient media access control in wireless sensor networks," in SenSys '07: Proceedings of the 5th international conference on Embedded networked sensor systems, (New York, NY, USA), pp. 59-72, ACM, 2007. 